\title{
Avaliação da incidência e fatores de risco da retenção de placenta em vacas mestiças leiteiras
}

[Evaluation of incidence rate and risk factors of retained placenta of crossbred dairy cattle]

\author{
M.M. Nobre', S.G. Coelho², J.P.A. Haddad ${ }^{2}$, E.F. Campos $^{3}$, A.M.Q. Lana ${ }^{2}$, \\ R.B. Reis ${ }^{2}$, H.M. Saturnino ${ }^{2}$ \\ ${ }^{1}$ Embrapa Gado de Leite - Juiz de Fora, MG \\ ${ }^{2}$ Escola de Veterinária - Universidade Federal de Minas Gerais - Belo Horizonte, MG \\ ${ }^{3}$ ReHagro - Belo Horizonte, MG.
}

\begin{abstract}
RESUMO
Avaliaram-se a incidência, os fatores de risco e o impacto da retenção de placenta tanto no desempenho reprodutivo quanto no produtivo de vacas mestiças leiteiras, considerando-se: ano e época de parição, ordem de lactação, escore de condição corporal (ECC), duração da gestação, tipo de parto e número e sexo dos bezerros. Utilizaram-se primíparas e multíparas, com e sem retenção de placenta, na época de chuva e de seca, para estudar: período de serviço, número de doses de sêmen/concepção e produção de leite em até 305 dias e no pico da lactação. A incidência da retenção foi de 12,8\%. Os fatores de risco da retenção de placenta foram: período de chuvas, ordem de lactação - segunda, terceira e acima da quarta -, ECC abaixo de 3,5 e acima de 4,0 - aborto, natimorto, prematuro, parto auxiliado e parto gemelar. Verificou-se aumento de 51,2 e 27,5 dias no período de serviço e aumento de 1,2 e 0,6 no número de doses de sêmen, em multíparas com retenção de placenta que pariram no período de chuva ou seca, respectivamente $(\mathrm{P}<0,05)$.
\end{abstract}

Palavras-chave: bovino de leite, período de serviço, produção de leite

\begin{abstract}
The incidence rate, risk factors and impact of retained placenta on the reproductive and productive performance of crossbred dairy cows was evaluated. The frequency distribution of retained placenta and also a Poisson multivariate analysis were developed for the following variables: year, calving season, lactation order, body condition score, gestation length, type of delivery, number and sex of calves. First calf heifers and multiparous cows, with and without retained placenta, during the rainy and dry season, had their average results compared in the following items: days from calving to first service, number of semen doses per conception, milk production up to 305 days and lactation peak. The incidence rate of retained placenta was $12.8 \%$. The risk factors were: rainy calving season, $2^{\text {nd }}, 3^{\text {rd }}$ and above $4^{\text {th }}$ lactation orders, body scores below 3.5 and greater than 4.0, abortion, stillbirth, premature birth, assisted birth and multiple birth. An increase of 51.2 and 27.5 days in the average interval between calving and first service, and of 1.2 and 0.6 in the average number of semen doses was observed in multiparous cows with retained placenta, giving birth during the rainy and dry seasons, respectively $(P<0.05)$.
\end{abstract}

Keywords: dairy cattle, calving to first service, milk production

\section{INTRODUÇÃO}

A liberação da placenta após o parto envolve a perda da adesão materno-fetal e ocorre somente após a maturação do placentoma, sendo que as

Recebido em 10 de agosto de 2010

Aceito em 7 de novembro de 2011

*Autor para correspondência (corresponding author)

E-mail: sandragesteiracoelho@gmail.com contrações uterinas ajudam mecanicamente essa liberação que ocorre fisiologicamente entre três e seis horas após o parto (Meça et al., 2006). Kelton et al. (1998) caracterizaram a retenção de placenta como falha da expulsão da placenta dentro de 24 horas após o parto. 
Jordan e Fourdraine (1993) verificaram que a incidência de retenção de placenta em 61 rebanhos nos Estados Unidos foi de 9,0\%. A incidência aparentemente varia de acordo com o ano, estação do ano e rebanho com causas ainda não compreendidas. Além disso, várias condições podem estar associadas à retenção de placenta, como, por exemplo, abortos, doenças, hipocalcemia, parto distocíaco, partos gemelares, duração da gestação, idade, estação do ano e nutrição (Muller e Owens, 1974). A idade da vaca e fatores específicos de cada propriedade são obviamente importantes para a manifestação da condição e para seu impacto sobre a produção e a reprodução (Wiltbank, 2006). Segundo Rajala et al. (1998), a retenção de placenta pode afetar a eficiência reprodutiva e a produção de leite dos animais.

O objetivo deste trabalho foi avaliar a incidência de retenção de placenta e a associação entre fatores de risco e retenção de placenta em uma propriedade leiteira, assim como o impacto dessa afecção tanto no desempenho reprodutivo quanto no produtivo.

\section{MATERIAL E MÉTODOS}

Avaliou-se o banco de dados de uma fazenda localizada no estado de Minas Gerais, contendo informação de partos ocorridos de janeiro de 2008 a março de 2010. A fazenda, com 900 vacas em lactação, de composição genética de 3/4 a 31/32 Holandês Zebu, produz em torno de 20.000 litros/ha/ano em sistema de semiconfinamento, com média total de $5.399 \mathrm{~kg}$ em 305 dias de lactação.

Foram elaboradas tabelas de contingência para verificação da distribuição de frequência dos casos de retenção de placenta para as seguintes variáveis: ano 2008 - 2009 e 2010 -; época de parição - chuva e seca -; ordem de lactação primeira, segunda, terceira e acima da quarta ordem -; escore de condição corporal ao parto escala de 1 a 5 pontos segundo Edmonson et al. (1989) -; duração da gestação - curta, normal e longa -; tipo de parto - normal, auxiliado, aborto, natimorto e prematuro -; número de bezerros nascidos ao parto - simples e gemelar; e sexo da cria - macho e fêmea. Foram obtidas equações de regressão usando-se o Modelo de Poisson para o estudo de possíveis associações dos fatores de risco e para determinar a contribuição de cada variável no aumento ou na diminuição da incidência de retenção.

Primíparas e multíparas, com e sem retenção de placenta, no período de chuva e seca, foram incluídas no estudo do período de serviço, número de doses de sêmen por concepção, produção de leite em até 305 dias e produção no pico da lactação. O diagnóstico de gestação foi realizado por palpação retal 60 dias após a inseminação artificial. $\mathrm{O}$ diagnóstico de retenção de placenta foi definido como a presença da placenta 24 horas após o parto.

A pesagem de leite foi realizada a cada 15 dias. Para avaliar as diferenças entre os grupos, foi realizada análise de variância não paramétrica utilizando-se o teste estatístico de KruskallWallis e o teste de Mann-Whitney $(\mathrm{P}<0,05)$

\section{RESULTADOS E DISCUSSÃO}

Os valores de dispersão de frequência apresentados na Tab. 1 indicam associações entre todas as variáveis $(\mathrm{P}<0,05)$.

A incidência de retenção de placenta foi de $12,8 \%$. Este valor é mais alto que os apresentados na Nova Zelândia $(2,0 \%)$, GrãBretanha $(3,8 \%)$, Irlanda $(4,1 \%)$, Arábia Saudita (6,3\%), Estados Unidos (7,7\%), Suécia $(7,7 \%)$, Israel $(8,4 \%)$ e Índia $(8,9 \%)$, e mais baixo que os obtidos em Bangladesh $(39,0 \%)$, Indonésia $(30,0 \%)$ e Tunísia $(15,0 \%)$, de acordo com Laven e Peters (1996).

As variações de incidência entre países ocorrem possivelmente em razão do diagnóstico utilizado - momento em que a placenta é considerada retida - assim como do sistema de produção. Segundo Laven e Peters (1996), as taxas de retenção de placenta são maiores em sistemas de pastejo tropical, em que os animais estão mais sujeitos a estresse térmico e nutricional. 
Avaliação da incidência...

Tabela 1. Distribuição de frequência da retenção de placenta em vacas mestiças leiteiras de acordo com cada variável

\begin{tabular}{|c|c|c|c|}
\hline \multirow[t]{2}{*}{ Variável* } & \multicolumn{2}{|c|}{ Retenção de placenta } & \multirow[b]{2}{*}{ Total } \\
\hline & Sim & Não & \\
\hline \multicolumn{4}{|l|}{ Ano } \\
\hline 2008 & $122(13,4 \%)$ & $792(86,7 \%)$ & 914 \\
\hline 2009 & $105(11,3 \%)$ & $823(88,7 \%)$ & 928 \\
\hline 2010 & $26(18,6 \%)$ & $114(81,4 \%)$ & 140 \\
\hline Todos os anos & $253(12,8 \%)$ & $1729(87,2 \%)$ & 1982 \\
\hline \multicolumn{4}{|c|}{ Época de parição } \\
\hline Chuva & $149(17,3 \%)$ & $713(82,7 \%)$ & 862 \\
\hline Seca & $104(9,3 \%)$ & $1.016(90,7 \%)$ & 1.120 \\
\hline \multicolumn{4}{|c|}{ Ordem de lactação } \\
\hline $1^{\mathrm{a}}$ & $62(9,1 \%)$ & $622(90,9 \%)$ & 684 \\
\hline $2^{a}$ & $76(14,4 \%)$ & $452(85,6 \%)$ & 528 \\
\hline $3^{\mathrm{a}}$ & $49(13,4 \%)$ & $317(86,6 \%)$ & 366 \\
\hline Acima da $4^{\mathrm{a}}$ & $66(16,3 \%)$ & $338(83,7 \%)$ & 404 \\
\hline \multicolumn{4}{|l|}{ Escore corporal } \\
\hline 2,0 & $6(42,9 \%)$ & $8(57,1 \%)$ & 14 \\
\hline 2,5 & $33(26,2 \%)$ & $93(73,8 \%)$ & 126 \\
\hline 3,0 & $118(16,1 \%)$ & $614(83,9 \%)$ & 732 \\
\hline 3,5 & $45(6,7 \%)$ & $630(93,3 \%)$ & 675 \\
\hline 4,0 & $7(7,6 \%)$ & $85(92,4 \%)$ & 92 \\
\hline 4,5 & $6(40,0 \%)$ & $9(60,0 \%)$ & 15 \\
\hline \multicolumn{4}{|c|}{ Duração da gestação } \\
\hline Curta & $104(32,8 \%)$ & $213(67,2 \%)$ & 317 \\
\hline Normal & $147(9,0 \%)$ & $1.493(91,0 \%)$ & 1.640 \\
\hline Longa & $2(8,0 \%)$ & $23(92,0 \%)$ & 25 \\
\hline \multicolumn{4}{|l|}{ Tipo de parto } \\
\hline Normal & $122(7,9 \%)$ & $1.416(92,1 \%)$ & 1.538 \\
\hline Auxiliado & $10(22,2 \%)$ & $35(77,8 \%)$ & 45 \\
\hline Natimorto & $26(24,5 \%)$ & $80(75,5 \%)$ & 106 \\
\hline Prematuro & $68(27,4 \%)$ & $180(72,6 \%)$ & 248 \\
\hline Aborto & $27(60,0 \%)$ & $18(40,0 \%)$ & 45 \\
\hline \multicolumn{4}{|c|}{ Número de bezerros } \\
\hline Simples & $228(11,8 \%)$ & $1.706(88,2 \%)$ & 1.934 \\
\hline Gemelar & $25(52,1 \%)$ & $23(47,9 \%)$ & 48 \\
\hline \multicolumn{4}{|l|}{ Sexo da cria } \\
\hline Fêmea & $103(10,8 \%)$ & $852(89,2 \%)$ & 955 \\
\hline Macho & $133(13,9 \%)$ & $826(86,1 \%)$ & 959 \\
\hline
\end{tabular}

*Associação em cada variável pelo teste do qui-quadrado $(\mathrm{P}<0,05)$.

Os resultados da análise multivariada são apresentados na Tab. 2, sendo que os coeficientes de risco negativos indicam fatores de proteção para o rebanho, ou seja, variáveis que contribuem para redução da ocorrência de retenção de placenta, enquanto os valores positivos indicam fatores de risco para a afecção.

Na parição no período de chuvas, o coeficiente foi positivo, indicando ser importante fator de risco na incidência de retenção de placenta. $\mathrm{O}$ estresse térmico e de ambiente, nesse período, associado a possíveis deficiências nutricionais ocasionadas pelo menor consumo de alimentos compromete a manutenção do sistema imune, resultando em aumento da susceptibilidade a doenças, entre elas, a retenção de placenta. Segundo Wiltbank (2006), há maior probabilidade de aumento da taxa de retenção de placenta quando as vacas são expostas a estresse intenso em razão do aumento do cortisol sérico no lado materno da circulação, inibindo a rejeição da placenta pelo sistema imune. 
Tabela 2. Coeficientes de risco e proteção na incidência de retenção de placenta em vacas mestiças leiteiras

\begin{tabular}{lccccc}
\hline Variável* $^{*}$ & Coeficiente & $\begin{array}{c}\text { Erro } \\
\text { padrão }\end{array}$ & $\begin{array}{c}\text { Valor de } \\
\mathrm{p}\end{array}$ & $\begin{array}{c}\text { Limite } \\
\text { inferior }\end{array}$ & $\begin{array}{c}\text { Limite } \\
\text { superior }\end{array}$ \\
\hline Ano 2009 & $-0,380$ & 0,134 & 0,004 & $-0,672$ & $-0,088$ \\
Ano 2010 & $-0,203$ & 0,202 & 0,317 & $-0,702$ & 0,297 \\
Época de parição (chuva) & 0,329 & 0,132 & 0,012 & 0,040 & 0,619 \\
Segunda parição & 0,666 & 0,170 & $<0,001$ & 0,285 & 1,047 \\
Terceira parição & 0,711 & 0,193 & $<0,001$ & 0,290 & 1,132 \\
Acima da quarta parição & 0,893 & 0,177 & $<0,001$ & 0,503 & 1,283 \\
Escore corporal & $-0,593$ & 0,169 & $<0,001$ & $-0,933$ & $-0,252$ \\
Parto auxiliado & 0,911 & 0,265 & 0,001 & 0,220 & 1,602 \\
Parto prematuro & 1,149 & 0,145 & $<0,001$ & 0,820 & 1,478 \\
Natimorto & 1,005 & 0,210 & $<0,001$ & 0,532 & 1,478 \\
Aborto & 1,878 & 0,216 & $<0,001$ & 1,273 & 2,484 \\
Parto gemelar & 0,745 & 0,163 & $<0,001$ & 0,286 & 1,205 \\
Constante & $-1,141$ & 0,574 & 0,047 & $-2,299$ & 0,016 \\
*Variáveis significativas pelo modelo de regressão de Poisson $(\mathrm{P}<0,05)$ & & & &
\end{tabular}

No ano de $2008,42 \%$ dos partos ocorreram no período das águas, e em 2009, esse valor foi de $36,4 \%$. Essa redução no número de partos durante a estação chuvosa foi relacionada a mudanças no manejo da propriedade com concentração de partos no outono, sem chuva e barro nas áreas de circulação e próximo aos cochos, refletindo em melhores condições sanitárias e nutricionais no período de transição, que seguramente contribuíram para a redução dos casos de retenção de placenta. Oltenacu et al. (1990) verificaram tendência de menor risco de incidência de retenção de placenta nos partos no inverno quando comparados a partos no verão em rebanhos da Suécia.

Verificaram-se coeficientes de risco positivos para a ordem de lactação, ou seja, o número estimado da frequência da retenção de placenta aumenta à medida que se aumenta a ordem da lactação. É plausível admitir que as multíparas apresentem sistema imune menos efetivo em responder aos desafios atribuídos ao desgaste de lactações anteriores, o que poderia justificar a maior ocorrência de retenção de placenta em animais mais velhos. $\mathrm{O}$ aumento da incidência da retenção de placenta à medida que aumenta a idade da vaca também foi verificado por Van Werven et al. (1992).

Verificou-se coeficiente negativo para escore corporal (ECC), sendo, portanto, um fator de proteção para o rebanho. De acordo com Tab. 1, as menores taxas de incidência ocorreram em animais que pariram com escores 3,5 e 4,0, comprovando a importância dessa meta para a propriedade. Neste estudo, $46 \%$ dos animais apresentaram escore 3,5 e 4,0 , e $44 \%$ apresentaram escore 3,0 , o que justificaria melhora no manejo da propriedade para mudar a classificação de 3,0 para 3,5. A maior incidência de retenção de placenta verificada nos animais com escore 2,0 e 2,5 pode estar relacionada ao sistema imune debilitado que aumenta a susceptibilidade a doenças. Já para o escore 4,5, a provável justificativa seria a maior probabilidade de ocorrência de distúrbios metabólicos.

Verificou-se que tipo de parto, exceto o normal, apresentou os maiores coeficientes de risco no modelo multivariável, que resultou em maior impacto no aumento da incidência da retenção de placenta $(\mathrm{P}<0,001)$. Grunert e Birgel (1982) observaram que, em abortos e partos prematuros, os placentomas estão imaturos em razão da diminuição do período de gestação, que ocasiona falha da separação imediata da junção cotilédone-carúncula. Em partos auxiliados, talvez possa existir relação entre a manipulação do bezerro e o aumento do edema local. O edema das vilosidades coriônicas causado por distocias foi citado por Grunert e Birgel (1982) como importante causa de retenção da placenta atribuída à forte adesão das membranas fetais. Além disso, o parto auxiliado provavelmente irá gerar dor e, nesse caso, as respostas ao estresse com liberação de endorfinas e catecolaminas podem reduzir a motilidade uterina, apesar de não ser esta a causa principal de retenção de 
placenta segundo Santos (2010). Erb et al. (1958) relataram maior incidência de retenção de placenta associada a aborto e natimorto.

Dos 48 partos gemelares, 15 foram nascimentos de duas fêmeas, sete de dois machos e 26 de uma fêmea e um macho. Além disso, 38\% desses partos ocorreram com período de gestação curto e $62 \%$ normal e longa. Já em relação ao tipo de parto, 60\% dos partos gemelares apresentaram alguma alteração (aborto, auxiliado, antimorto um prematuro) e $40 \%$ foi parto normal. O parto gemelar também foi fator predisponente no aumento da incidência da retenção de placenta. Erb et al. (1958) e Muller e Owens (1974) também verificaram maiores taxas de incidência de retenção de placenta em partos gemelares provavelmente relacionados à distocia.

No modelo de Poisson, a duração da gestação não apresentou significância perante as demais variáveis, provavelmente devido ao fato de a gestação curta estar diretamente relacionada com abortos e partos prematuros, que são fatores de risco para a retenção de placenta. Grunert e Birgel (1982) verificaram que o encurtamento do período de gestação está relacionado com a imaturidade dos placentomas que normalmente ocorre de três a cinco dias antes do parto com duração normal da gestação. $O$ processo de maturação é essencial para a liberação da placenta. Muller e Owens (1974) e Wheterill (1965) também observaram que o período de gestação mais curto aumenta a incidência de retenção de placenta. Em gestações longas, Wheterill (1965) verificou maior incidência, porém a característica foi a gestação com duração acima de 285 dias.

O sexo da cria não apresentou significância perante as demais variáveis no modelo multivariável, apesar da associação verificada no teste do qui-quadrado. Provavelmente essa perda de significância ocorreu pela existência de outros efeitos que estavam influenciando a taxa de incidência, como, por exemplo, parição nas chuvas, aborto, parto prematuro, natimorto, parto gemelar e ECC menor que 3,5 e maior que 4,5.

$\mathrm{Na}$ Tab. 3, apresentam-se os contrastes significativos referentes ao impacto no desempenho reprodutivo de primíparas e multíparas com e sem retenção de placenta e parição nos períodos de chuva e seca.

Tabela 3. Desempenho reprodutivo de primíparas e multíparas parindo nos períodos de chuva e seca com presença ou ausência de retenção de placenta em vacas mestiças leiteiras

\begin{tabular}{lcccc}
\hline \multirow{2}{*}{ Grupo } & $\mathrm{N}$ & \multicolumn{3}{c}{ Desempenho reprodutivo } \\
\cline { 3 - 5 } & & $\begin{array}{c}\text { Período de serviço } \\
\text { (dias) }\end{array}$ & $\begin{array}{c}\text { Número de doses } \\
\text { de sêmen }\end{array}$ & $\begin{array}{c}\text { Contrastes } \\
\text { (valor de P) }\end{array}$ \\
\hline Grupo 1: RP-*, Primípara, Chuva & 149 & 116,4 & 1,8 & \\
Grupo 2: RP-, Multípara, Chuva & 285 & 126,7 & 2,1 & $2 \times 6(\mathrm{P}<0,001)$ \\
Grupo 3: RP-, Primípara, Seca & 310 & 104,8 & 2,1 & \\
Grupo 4: RP-, Multípara, Seca & 468 & 102,1 & 2,0 & $4 \times 8$ (P<0,05) \\
Grupo 5: RP+**, Primípara, Chuva & 24 & 137,3 & 2,1 & \\
Grupo 6: RP+, Multípara, Chuva & 59 & 177,9 & 3,3 & \\
Grupo 7: RP+, Primípara, Seca & 14 & 99,1 & 1,9 & \\
Grupo 8: RP+, Multípara, Seca & 49 & 129,6 & 2,6 & \\
Todos os grupos & 1358 & 114,3 & 2,1 & \\
\hline
\end{tabular}

*RP-: ausência de retenção de placenta após 24 horas do parto.

**RP+: presença de retenção de placenta após 24 horas do parto.

Testes de Kruskall-Wallis e Mann-Whitney.

Verificou-se aumento de 51,2 e 27,5 dias no período de serviço e de 1,2 e 0,6 doses de sêmen por concepção de multíparas com retenção de placenta parindo nos períodos de chuva e seca, respectivamente, quando comparadas com multíparas sem retenção de placenta $(\mathrm{P}<0,05)$. Nas primíparas, o período de serviço não foi afetado pela retenção de placenta $(\mathrm{P}>0,05)$. Talvez essa diferença entre a retenção de placenta afetar o período de serviço entre multíparas e primíparas seja devido ao aumento de desafio da produção de leite das multíparas, o que afeta possivelmente o sistema imune desse grupo de animais, levando maior tempo de recuperação da doença ou predispondo o animal a outras afecções. Ainda, segundo Van Werven (1992), as primíparas apresentam involução uterina mais rápida, melhores condições de 
endométrio e sistema imune mais eficiente, sendo esses fatores responsáveis pelos menores efeitos da retenção de placenta no desempenho reprodutivo dessa categoria.

Martin et al. (1986) e Etherington et al. (1985) também verificaram aumento no período médio de serviço de multíparas. Van Werven et al. (1992) relataram aumento somente em vacas acima da quarta ordem de parição, e não foi observado efeito prejudicial da retenção de placenta sobre o período de serviço de primíparas. Muller e Owens (1974) e Pelissier (1972) também verificaram aumento no número de serviços por concepção em vacas com retenção de placenta.

O maior impacto verificado no período de serviço de multíparas que pariram na época de chuva pode ser explicado pelo maior desafio enfrentado nesse período do ano no que diz respeito ao estresse térmico e ao conforto ambiental, causando redução no consumo de alimentos e, consequentemente, atraso no retorno à atividade cíclica ovariana. Segundo Wiltbank (2006), a maioria dos estudos demonstra que vacas mais velhas geralmente têm mais problemas reprodutivos após a retenção de placenta.

Apesar da diferença verificada no período de serviço de multíparas, alguns autores acreditam que o efeito direto na fertilidade somente ocorre quando a retenção de placenta aparece associada ao quadro de metrite. Segundo Artunduaga et al. (2008), um dos principais objetivos da pecuária leiteira é o de obter, anualmente, um parto por vaca e, para isso, é necessário o conhecimento de fatores de risco, para a implantação de medidas preventivas visando à diminuição da incidência da retenção de placenta.

$\mathrm{Na}$ Tab. 4, observa-se que não houve diferença na produção em até 305 dias e no pico de produção de leite de primíparas e multíparas, com e sem retenção de placenta nas diferentes épocas de parição.

Tabela 4. Desempenho produtivo de primíparas e multíparas parindo nos períodos de chuva e seca com presença ou ausência de retenção de placenta em vacas mestiças leiteiras

\begin{tabular}{lccc}
\hline \multirow{2}{*}{ Grupo } & \multicolumn{3}{c}{ Desempenho produtivo } \\
\cline { 2 - 4 } & $\mathrm{N}$ & $\begin{array}{c}\text { Produção em até 305 } \\
\text { dias (litros) }\end{array}$ & $\begin{array}{c}\text { Pico de produção } \\
\text { de leite (litros) }\end{array}$ \\
\hline Grupo 1: RP-*, Primípara, Chuva & 126 & 5352,8 & 26,5 \\
Grupo 2: RP-, Multípara, Chuva & 227 & 6331,4 & 31,3 \\
Grupo 3: RP-, Primípara, Seca & 192 & 4210,2 & 24,2 \\
Grupo 4: RP-, Multípara, Seca & 257 & 5493,1 & 30,7 \\
Grupo 5: RP+**, Primípara, Chuva & 19 & 4972,4 & 26,0 \\
Grupo 6: RP+, Multípara, Chuva & 45 & 6030,0 & 30,0 \\
Grupo 7: RP+, Primípara, Seca & 7 & 3745,4 & 22,6 \\
Grupo 8: RP+, Multípara, Seca & 32 & 5096,0 & 29,8 \\
Todos os grupos & 905 & 5399,9 & 28,6 \\
\hline
\end{tabular}

*RP-: ausência de retenção de placenta após 24 horas do parto.

**RP+: presença de retenção de placenta após 24 horas do parto.

Testes de Kruskall-Wallis e Mann-Whitney.

O impacto da redução da produção de leite talvez tenha ocorrido apenas nos primeiros dias da lactação, não sendo suficiente para comprometer o pico e a produção total de leite. Essa hipótese está de acordo com os resultados obtidos por Rajala et al. (1998), que constataram efeito de curto prazo, mas não constataram efeito da retenção de placenta sobre a produção de leite aos 305 dias.

\section{CONCLUSÕES}

O conhecimento da incidência da retenção de placenta, assim como a associação dos fatores de risco, permite a elaboração de medidas direcionadas e eficazes de prevenção da afecção no rebanho. A retenção de placenta não afeta a produção de leite, porém apresenta efeito deletério sobre eficiência reprodutiva, sendo que índices adequados de eficiência de produção 
estão diretamente relacionados à sustentabilidade econômica dos sistemas de produção leiteira.

\section{REFERÊNCIAS}

ARTUNDUAGA, M.A.T.; SÁ FORTE, R.V.; COELHO, S.G. et al. Atividade ovariana de vacas leiteiras em dietas com propilenoglicol ou monensina no período de transição. Arq. Bras. Med. Vet. Zootec., v.60, p.289-293, 2008.

EDMONSON, A.J.; LEAN, I.J.; WEAVER, L.D. et al. A body condition scoring chart for Holstein dairy cows. J. Dairy Sci., v.72, p.68-78, 1989.

ERB, R.E.; HINZE, P.M.; GILDOW, E.M. et al. Retained fetal membranes - the effect on prolificacy of dairy cattle. J. Am. Vet. Med. Assoc., v.133, p.489-496, 1958.

ETHERINGTON, W.G.; MARTIN, S.W.; DOHOO, I.R. et al. Interrelationships between ambient temperature, age at calving, postpartum reproductive events and reproductive performance in dairy cows: a path analysis. Can. J. Comp. Med., v.49, p.254-260, 1985.

GRUNERT, E.; BIRGEL, E.H. Obstetrícia Veterinária. Porto Alegre: Sulina, 1982. 323 p.

JORDAN, E.R.; FOURDRAINE, R.H. Characterization of the management practices of the top milk producing herds in the country. $J$. Dairy Sci., v.76, p.3247-3256, 1993.

KELTON, D.F.; LISSEMORE, K.D.; MARTIN, R.E. Recommendations for recording and calculating the incidence of selected clinical diseases of dairy cattle. J. Dairy Sci., v.81, p.2502-2509, 1998.

LAVEN, R.A.; PETERS, A.R. Bovine retained placenta: aetiology, pathogenesis and economic loss. Vet. Rec., v.139, p.465-471, 1996.

MARTIN, J.M.; WILCOX, C.J.; MOYA, J. et al. Effects of retained fetal membranes on milk yield and reproductive performance. J. Dairy Sci., v.69, p.1166-1168, 1986.
MEÇA, K.K.O.; VASCONCELOS, A.C., MORO, L. Inibição de apoptose e retardo da maturação placentária: um provável mecanismo da retenção de placentária na brucelose bovina (revisão de literatura). Biosci. J., v.22, p.163174, 2006.

MULLER, L.D.; OWENS, M.J. Factors associated with the incidence of retained placenta. J. Dairy Sci., v.57, p.725-728, 1974.

OLTENACU, P.A.; FRICK, A.; LINDHE, B. Epidemiological study of several clinical diseases, reproductive performance and culling in primiparous Swedish cattle. Prev. Vet. Med., v.9, p.59-74, 1990.

PELISSIER, C.L. Herd breeding problems and their consequences. J. Dairy Sci., v.55, p.385391, 1972.

RAJALA, P.J.; GROHN, Y.T. Effects of dystocia, retained placenta, and metritis on milk yield in dairy cows. J. Dairy Sci., v.81, p.31723181, 1998.

SANTOS, J.E.P. Doenças uterinas em vacas de leite: prevalência, fatores de risco e tratamento. In: CURSO NOVOS ENFOQUES NA PRODUÇÃO E REPRODUÇÃO DE BOVINOS, 14., 2010, Uberlândia. Anais... Uberlândia: 2010. p.393410.

VAN WERVEN, T.; SCHUKKEN, Y.H.; LLOYD, J. et al. The effects of duration of retained placenta on reproduction, milk production, postpartum disease and culling rate. Theriogenology, v.37, p.1191-1203, 1992.

WHETERILL, G.D., Retained placenta in the bovine: a brief review. Can. Vet. J., v.6, p.290294, 1965.

WILTBANK, M. C. Prevenção e tratamento da retenção de placenta. In: CURSO NOVOS ENFOQUES NA PRODUÇÃO E REPRODUÇÃO DE BOVINOS, 10., 2006, Uberlândia. Anais... Uberlândia, 2006. p.61-70. 